\title{
Discrete Wavelet Transform and Cross Bilateral Filter based Image Fusion
}

\author{
Sonam \\ Department of Computer Science, Babasaheb Bhimrao Ambedkar University, Lucknow-226025, India \\ E-mail: sonam870115@g mail.com \\ Manoj Kumar \\ Department of Computer Science, Babasaheb Bhimrao Ambedkar University, Lucknow-226025, India \\ E-mail: mkjnuiitr@gmail.com
}

\begin{abstract}
The main objective of image fusion is to obtain an enhanced image with more relevant information by integrating complimentary information from two source images. In this paper, a novel image fusion algorithm based on discrete wavelet transform (DWT) and cross bilateral filter $(\mathrm{CBF})$ is proposed. In the proposed framework, source images are decomposed into low and high frequency subbands using DWT. The low frequency subbands of the transformed images are combined using pixel averag ing method. Meanwhile, the high frequency subbands of the transformed images are fused with weighted average fusion rule where, the weights are computed using $\mathrm{CBF}$ on both the images. Finally, to reconstruct the fused image inverse DWT is performed over the fused coefficients. The proposed method has been extensively tested on several pairs of multi-focus and multisensor images. To compare the results of proposed method with different existing methods, a variety of image fusion quality metrics are employed for the qualitative measurement. The analysis of comparison results demonstrates that the proposed method exhibits better results than many other fusion methods, qualitatively as well as quantitatively.
\end{abstract}

Index Terms-Image Fusion, Discrete Wavelet Transform, Cross Bilateral Filter, Standard Deviation, Correlation Coefficients.

\section{INTRODUCTION}

Nowadays, image fusion has gained much attention in image processing and computer vision. Image fusion is the process of combining the significant visual information from various source images of the same scene or view to produce a single enhanced image without any distortion or less information in images. The obtained fused image is more useful for human visual perception and further image processing operations [1]. Image fusion has been used in many disparate fields such as, medical imaging [2,3], military [4], surveillance [5], robotics and remote sensing [6], etc.

Generally, an image having less information does not provide an appropriate analysis of the scene. Consider, two images where one image is focused on some part of the scene and rest of part is in out-of-focus and another image is focused on the portion which is defocused in the first image however, defocused region which is focused in the first image. To get the entire scene in focus in a single image is a difficult task. Therefore, by integrating the relevant details from both the source images, a fused image with complete information can be obtained. This whole process of image fusion is called as multi-focus image fusion and these source images are known as multi-focus images.

The images captured from different sensors are mu ltisensor images such as, CT (Computed Tomography) and MRI (Magnetic Resonance Imaging). These images may provide a different kind of information of the same organ to diagnose the diseases. CT images give the information of bone, blood vessels and hard tissues, whereas, MRI images give the information of the soft tissues. These two different images individually provide the different information according to their sensor ability but if we combined these images into a single image then all the relevant information (bone and soft tissues) of the organ can be presented in a single image which may provide more beneficiaries for diagnosis purpose. The fusion of multisensor images is known as the multisensor image fusion.

Image fusion process can be conducted at different levels, depending on represented information and applications. These levels are categorized into signal or pixel level, object or feature level and symbol or decision level [7]. In pixel level image fusion, the visual information of the source images is fused with their respective pixels to generate a single fused image. This level represents the lowest level of fusion. Feature level fusion defines the process of combining the features such as, edges, texture or color that have already been extracted from the source images. Finally, the decision level fusion represents the highest level of fusion, which combines the results from multiple algorith ms to obtain a final fused image. Among these, pixel level fusion is widely used in most image fusion applications due to the advantage of containing original information, easy implementation and low time consumption.

Further, fusion methods can be categorized into two domains. One is the spatial domain based methods and 
the other is the transform domain based methods [11]. The spatial domain methods perform the fusion process in spatial domain directly. The spatial domain methods are time efficient and easy to implement. On the other hand, the transform domain based methods use transformations like, discrete wavelet transform [8], stationary wavelet transform [12], dual tree complex wavelet transform [13], and so on. Recently, some other transform domain based fusion methods are also introduced, such as curvelet transform [14], contourlet transform [15], nonsubsampled contourlet transform (NSCT) [16], multi-resolution singular value decomposition (MSVD) [17] and so on.

The pixel by pixel averaging is the simplest image fusion method and due to this it has attracted many researchers in the last decades [8-10]. But this method suffers from a number of undesirable effects, such as reduced contrast. To address this problem, multiresolution analysis based methods have been proposed, which consist of the following three basic steps. First, the source images are decomposed into multi-resolution representations with low and high frequency information and corresponding transformed coefficients are obtained. Second, the obtained transformed coefficients are combined together according to some fusion rules. Finally, the inverse transform is performed over the fused coefficients to reconstruct the fused image [18]. Generally, multi-resolution based methods provide better results than the other transform methods. However, the other transformations also preserve the same salient features such as edges and lines and are used in fusion. For an instance, a discrete cosine transform (DCT) based image fusion is introduced for fusion instead of pyramids or wavelet [19]. Again, a new multi-resolution DCT decomposition based image fusion approach has been given to reduce the computational complexity without any loss of image information [10].

Many image fusion techniques have been developed such as, maximu m, minimu m, average methods, principal component analys is (PCA) [26,30,31], intensity-huesaturation [28]. In above discussed methods, average method provides the average information of the images but weighted average methods provide the information according to their weights and produce better fusion results. In [24], source images are fused by weighted average from the image details, where these details are extracted from the source images using CBF to improve the fusion performance. Hence, we proposed a novel pixel level image fusion scheme based on discrete wavelet transform and cross bilateral filter. The low frequency subbands are fused using pixel averaging method because approximation part contain most of the average information. Meanwhile, the high frequency subbands give the sharp details, therefore weighted average fusion rule is performed over the detail parts, where the weights of two different images contribute the information for fusion according to their weight value. The main contribution of this paper is to enhance the visual quality of the images by integrating all significant details of the source images using the pixel averaging and weighted average fusion rule.

The rest of this paper is organized as follows. In Section II, the basic theory of DWT and CBF are described. Proposed methodology is explained in Section III. Experimental results followed by discussion are presented in Section IV. Section V concludes the proposed work.

\section{BASIC THEORIES OF DWT AND CBF}

In this section, a brief review of the basic theories of discrete wavelet transform and cross bilateral filter is presented.

\section{A. Discrete Wavelet Transform}

Discrete wavelet transform contains the multiresolution analysis property, therefore it is widely used in image processing. It converts an image from spatial domain to frequency domain $[8,27,20]$. DWT decomposes the image into low and high frequency subbands. The low frequency subbands corresponds to approximation part, which contains average information of the entire image and is represented as (LL) subband. Whereas, the high frequency subbands are considered as detail parts containing the sharp information of images. The detail parts consist of three high frequency subbands (LH, HL and HH) as shown in Fig. 1 (a). For second level decomposition, only LL subband is further decomposed into four frequency subbands, whereas $\mathrm{LH}, \mathrm{HL}$ and $\mathrm{HH}$ subbands remain as such, as given in Fig. 1 (b). The decomposition levels can be increased as per the requirement.

A 2-D DWT [20] for image $f(x, y)$ of size $m \times n$ is defined as:

$$
\begin{aligned}
& W_{\phi}\left(j_{0}, u, v\right)=\frac{1}{\sqrt{m n}} \sum_{x=0}^{m-1} \sum_{y=0}^{n-1} f(x, y) \phi_{j_{0}, u, v}(x, y) \\
& W_{\psi}(j, u, v)=\frac{1}{\sqrt{m n}} \sum_{x=0}^{m-1} \sum_{y=0}^{n-1} f(x, y) \phi_{j, u, v}(x, y)
\end{aligned}
$$

where, Eqs. (1) and (2) are approximation and detail coefficients of image $f(x, y)$. Conversely, the image is reconstructed by performing inverse DWT. For the above given Eqs. (1) and (2), the inverse DWT is given as:

$$
\begin{aligned}
& f(x, y)=\frac{1}{\sqrt{m n}} \sum_{u} \sum_{v} W_{\varphi}\left(j_{0}, u, v\right) \varphi_{j_{0}, u, v}(x, y) \\
& +\frac{1}{\sqrt{m n}} \sum_{j=j_{0}}^{\infty} \sum_{u} \sum_{v} W_{\psi}(j, u, v) \psi_{j, u, v}(x, y)
\end{aligned}
$$

The DWT is applied over the image and the obtained results are shown in Fig. 2. Fig. 2 (a) shows the original lena image whereas, Fig. 2 (b) and 2 (c) show the first level decomposition and second level decomposition of the image after applying DWT. 


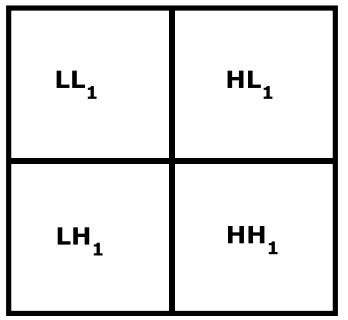

(a)

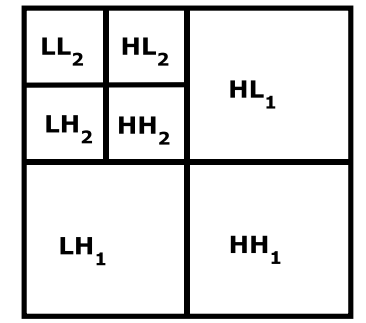

(b)
Fig.1. (a) First level DWT decomposition; (b) second level DWT decomposition.

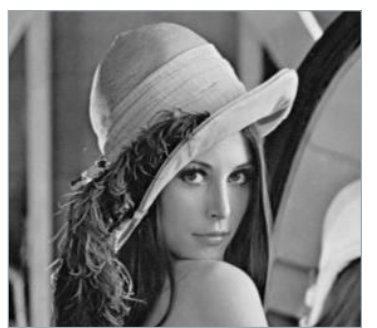

(a)

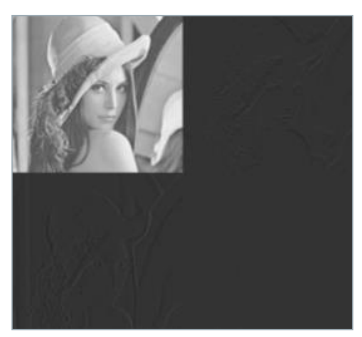

(b)

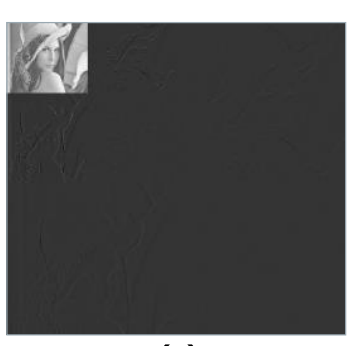

(c)
Fig.2. (a) Original lena image; (b) first level DWT decomposition; (c) second level DWT decomposition.

\section{B. Cross Bilateral Filter}

The Gaussian filter is the most popular filter which provides smoothness and removes noise present in the images. It is based on the weighted average of the intensity of the adjacent pixels. This filter removes the noise and provides smoothness but meantime also removes the sharp details. Therefore, to address this problem, the bilateral filter was introduced by Tomasi and Manduchi [21]. It is a local, nonlinear and noniterative filter which s moothes images while preserving edges and other sharp details. The bilateral filtering has been widely used in image processing applications. The spatial filter kernel is treated as a classical low pass filter used to obtain geometric closeness between the neighboring pixels, whereas the range filter kernel is treated like an edge-stopping function used for gray-level similarity between the neighboring pixels, which attenuates the filter kernel when the intensity differences between pixels are large. Both filter kernels are based on Gaussian distribution and the weights obtained from these filters depend not only on Euclidian distance but also on the distance in gray or color spaces. The bilateral filter $(\mathrm{BF})$ is the combination of spatial and range filters [2224].

For an image $X$, the output of bilateral filter at a pixel location $p$ is defined as:

$$
X_{f}(p)=\frac{1}{W} \sum_{\mathbf{q} \in S} G_{\sigma_{\mathrm{s}}}(\|p-q\|) \times G_{\sigma_{\mathrm{r}}}(|X(p)-X(q)|) X(q)
$$

where ,

$$
W=\sum_{q \in s} G_{\sigma_{s}}(\|p-q\|) G_{\sigma_{r}}(|X(p)-X(q)|)
$$

is a normalization factor, $\|p-q\|$ is the Euclidian distance between $p$ and $q$.

$$
G_{\sigma_{s}}(\|p-q\|)=e^{-\frac{\|p-q\|^{2}}{2 \sigma_{s}^{2}}}
$$

is a geometric closeness function and

$$
G_{\sigma_{r}}(|X(p)-X(q)|)=e^{-\frac{|X(p)-X(q)|^{2}}{2 \sigma_{r}^{2}}}
$$

is a gray-level similarity or edge-stopping function. $G_{\sigma_{s}}$ is a spatial Gaussian function that decreases symmetrically as the distance from the center increases. $G_{\sigma_{r}}$

is also a Gaussian function that decreases with the increase of the intensities difference between $X(p)$ and $X(q) . s$ is a spatial neighborhood of the pixel $p$. Parameters $\sigma_{s}$ and $\sigma_{r}$ are standard deviations of Gaussian functions $G_{\sigma_{s}}$ and $G_{\sigma_{r}}$ respectively and determine the amount of filtering for the image $X$.

Cross bilateral filter ( $\mathrm{CBF}$ ) considers both gray-level similarities and geometric closeness of neighboring pixels in image $X$ to shape the filter kernel and filters the image $Y$. The output of CBF [24,25] for image $Y$ at a pixel location $p$ is calculated as:

$$
Y_{C B F}(p)=\frac{1}{W} \sum_{\mathbf{q} \in S} G_{\sigma_{\mathrm{s}}}(\|p-q\|) \times G_{\sigma_{\mathrm{r}}}(|X(p)-X(q)|) Y(q)
$$

where,

$$
W=\sum_{q \in s} G_{\sigma_{s}}(\|p-q\|) G_{\sigma_{r}}(|X(p)-X(q)|)
$$

is a normalization factor and

$$
G_{\sigma_{r}}(|X(p)-X(q)|)=e^{-\frac{|X(p)-X(q)|^{2}}{2 \sigma_{r}^{2}}}
$$

is a gray-level similarity or edge-stopping function.

For images $X$ and $Y$, the detail image is obtained by subtracting $\mathrm{CBF}$ output from their respective source 
image and is given as $X_{D}=X-X_{C B F}$ and $Y_{D}=Y-Y_{C B F}$, respectively. These detail images are used to find the weights by measuring the strength of details as reported in [24].

\section{PRoposed METHEDOLOGY}

In this section, we briefly discuss the proposed method. In the proposed method, two images having less information are used to produce a single composite image with enhanced visual quality. Here, the two source images either multi-focus or multisensor are used for fusion. The DWT is performed over both the source images to decompose the images into low and high frequency subbands. The low frequency subbands, which is also called approximation part, contains the average information of the images. The low frequency subbands of both of the transformed images are fused using pixel averaging method to get the overall average information from both the source image in a single fused image. On the other hand, the high frequency subbands are called detail parts which contain the information of edges and sharp changes. The high frequency subbands of both of the transformed images are fused according to their weights in respective images to get the sharp information in the fused image. Thus, the fused image has overall average information as well as dominant sharp information chosen from both the source images. The block diagram of the proposed method is shown in Fig. 3.

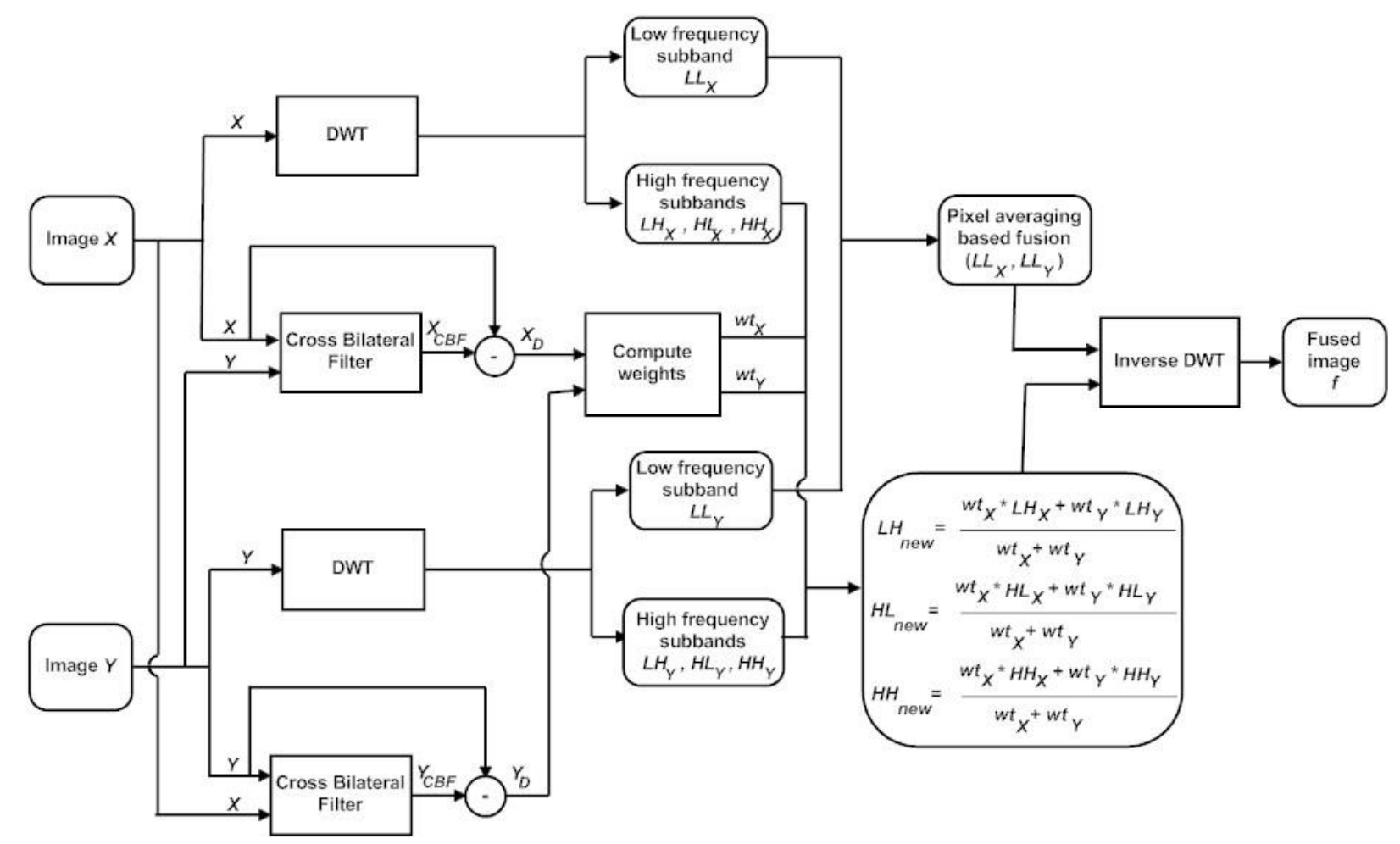

Fig.3. Block diagram of the proposed method

The steps of the proposed method are summarized in Table 1.

Table 1

\begin{tabular}{l}
\hline Algorithm: Proposed fusion method \\
\hline 1. Take two source images $X$ and $Y$. \\
2. Perform DWT over the images $X$ and $Y$ which \\
decomposes the images into low and high \\
frequency subbands. \\
3. Apply pixel averaging method to fuse the low \\
frequency subbands and obtain the average \\
fused coefficient ( $\left.L L_{\text {new }}\right)$. \\
Combine high frequency subbands by their \\
weighted average of both transformed images. \\
The weights are computed as in [24]:
\end{tabular}

a) Compute the covariance matrix as for both the images and then calculate horizontal and vertical detail strengths.

b) Integrate both detail strengths and obtain weight $w t_{X}$ and $w t_{Y}$ from images $X$ and $Y$.

c) Fuse the high frequency subbands with their respective weights using:

$$
H L_{\text {new }}=\frac{w t_{X} * H L_{X}+w t_{Y} * H L_{Y}}{w t_{X}+w t_{Y}}
$$

Similarly, we can obtain fused wavelet coefficients $\left(L H_{\text {new }}\right.$ and $\left.H H_{\text {new }}\right)$.

5. Perfom inverse DWT over the obtained fused coefficients ( $\mathrm{LL}_{\text {new }}, \mathrm{LH}_{\text {new }}, \mathrm{HL}_{\text {new }}$ and $\mathrm{HH}_{\text {new }}$ ) to reconstruct the fused image $\mathrm{f}$. 


\section{EXPERIMENT AL RESULTS AND DISCUSSION}

The proposed method is carried out on several pairs of multi-focus and multisensor images as shown in Fig. 4, 5, 6, 7 and 8. Each image is size of $512 \times 512$. Fig. 4 (a) and 4 (b) are blurred on left and right part images. Fig. 5 (a) and 5 (b) are upper side and lower side blurred images are called as dataset1. Fig. 6 (a) and 6 (b) are FLIR and LLTV images are called as dataset2. Dataset1 and dataset 2 images are taken from [17]. Fig. 7 (a) and 7 (b) are hoed images which are blurred on middle and boundary part. CT and MRI images of size $256 \times 256$ are shown in Fig. 8 (a) and 8 (b) are acquired from the link http://www.metapix.de/examples_r.htm. The parameters $\sigma_{s}=1.8$ and $\sigma_{r}=25$ are used for the proposed method with window size $5 \times 5$. The results of proposed methods are compared with some existing methods such as, DWT [8], PCA with DWT [26], SWT [12] and CBF [24] based fusion methods. The results obtained from above existing methods are shown in Fig. 4 (c-f), 5 (c-f), 6 (c-f), 7 (c-f) and 8 (c-f). However, the results obtained by proposed method are shown in Fig. $4(\mathrm{~g}), 5(\mathrm{~g}), 6(\mathrm{~g}), 7(\mathrm{~g})$ and 8 (g). Visually it can be seen that in most of the cases, the fused images obtained from proposed method are better than other existing methods.

But to evaluate the quality of fused image only visual inspection is not sufficient. Therefore, for quantitative measurement of the fused images some metrics are used. All the results of existing and proposed methods are tabulated in Table 2. It is found that in most of the cases the results of the proposed method have shown better performance than others and the best results are bolded. The metrics used in this paper are defined as:

\section{A. Average pixel intensity (API) or mean $(\mu)$}

It measures an index of contrast, which is defined as:

$$
A P I=\frac{\sum_{i=1}^{m} \sum_{j=1}^{n} f(i, j)}{m n}
$$

where, $f(i, j)$ represents fused image and the size of image is $m \times n$

\section{B. Standard deviation (SD)}

It is defined as:

$$
S D=\sqrt{\frac{\sum_{i=1}^{m} \sum_{j=1}^{n}(f(i, j)-\mu)^{2}}{m n}}
$$

It reflects the spread in data and higher value represents better fusion result.

\section{Average gradient $(A G)$}

It measures a degree of clarity and sharpness and is defined as:

$$
A G=\frac{\sum_{i=1}^{m} \sum_{j=1}^{n}\left((f(i, j)-f(i+1, j))^{2}+(f(i, j)-f(i, j+1))^{2}\right)^{1 / 2}}{m n}
$$

\section{Entropy (EN)}

It measures the amount of information presented in the fused image and is defined as:

$$
E N=-\sum_{k=0}^{255} p_{k} \log _{2}\left(p_{k}\right)
$$

where, $p_{k}$ is the probability of intensity value $k$ in image. The larger values denote better result.

\section{E. Mutual information (MI)}

It is defined as the sum of mutual information between source images and fused image and measures the degree of dependence of two images. The mutual information $M I_{X f}$ between source image $X$ and fused image $f$ is given as follows:

$$
M I_{X f}=\sum_{i, j} h_{X f}(i, j) \log _{2} \frac{h_{X f}(i, j)}{h_{X}(i) h_{f}(i, j)}
$$

where, $h_{X f}$ is the joint histogram between $X$ and $f$, respectively. Similarly, $M I_{Y f}$ represents the mutual information between source images $Y$ and fused image $f$. The total mutual information between source images $X, Y$ and fused image $f$ is defined as:

$$
M I_{\text {total }}=M I_{X f}+M I_{Y f}
$$

The higher $M I_{\text {total }}$ value implies better fusion results.

\section{F. Fusion symmetry (FS)}

It indicates how much symmetrical information of the fused image with respect to source image and is given as:

$$
F S=2-\left|\frac{M I_{X f}}{M I_{\text {total }}}-0.5\right|
$$

\section{G. Correlation coefficient $(C C)$}

It estimates a relevance of fused image to source image, larger value represents better fusion results which is defined as:

$$
C C=\left(r_{X f}+r_{Y f}\right) / 2
$$

where,

$$
r_{X f}=\frac{\sum_{i=1}^{m} \sum_{j=1}^{n}(X(i, j)-\bar{X})(f(i, j)-\mu)}{\sqrt{\left(\sum_{i=1}^{m} \sum_{j=1}^{n}(X(i, j)-\bar{X})^{2}\right)\left(\sum_{i=1}^{m} \sum_{j=1}^{n}(f(i, j)-\mu)^{2}\right)}}
$$


and

$$
r_{Y f}=\frac{\sum_{i=1}^{m} \sum_{j=1}^{n}(Y(i, j)-\bar{Y})(f(i, j)-\mu)}{\sqrt{\left(\sum_{i=1}^{m} \sum_{j=1}^{n}(Y(i, j)-\bar{Y})^{2}\right)\left(\sum_{i=1}^{m} \sum_{j=1}^{n}(f(i, j)-\mu)^{2}\right)}}
$$

\section{H. Spatialfrequency $(S F)$}

It estimates the overall information level in the regions of an image and is calculated as:

$$
S F=\sqrt{R F^{2}+C F^{2}}
$$

where, $R F$ and $C F$ are row and column frequency

$$
\begin{aligned}
& R F=\sqrt{\frac{\sum_{i=1}^{m} \sum_{j=1}^{n}(f(i, j)-f(i, j-1))^{2}}{m n}} \\
& C F=\sqrt{\frac{\sum_{i=1}^{m} \sum_{j=1}^{n}(f(i, j)-f(i-1, j))^{2}}{m n}}
\end{aligned}
$$

The large value of spatial frequency represents the large information in the image.

I. $Q^{X Y / f}$

It evaluates the total information transferred from the source images to fused image [29]. Mathematically, it is defined as:

$$
Q^{X Y / f}=\sum_{i=1}^{m} \sum_{j=1}^{n} \frac{\left[Q^{X f}(i, j) w^{X}(i, j)+Q^{Y f}(i, j) w^{Y}(i, j)\right]}{\sum_{i=1}^{m} \sum_{j=1}^{n}\left[w^{X}(i, j)+w^{Y}(i, j)\right]}
$$

where, $X, Y$ are source images and $f$ is fused image. The definitions of $Q^{X f}$ and $Q^{Y f}$ are same and given as:

$$
Q^{X f}(i, j)=Q_{g}^{X f}(i, j) \cdot Q_{\alpha}^{X f}(i, j)
$$

where $Q_{g}^{X f}(i, j)$ and $Q_{\alpha}^{X f}(i, j)$ represent the edge strength and orientation values at location $(i, j)$, respectively. The dynamic range for $Q^{X Y / f}$ is $[0,1]$ and it should be close to one for better fusion.

The other metrics $L^{X Y / f}, N^{X Y / f}$ and $N_{m}{ }^{X Y / f}$ are used to compute the total loss of information and noise or artifacts in fused image which are given in [9,24,29].

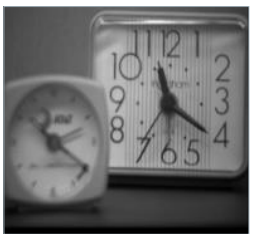

(a)

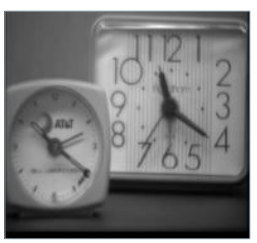

(d)

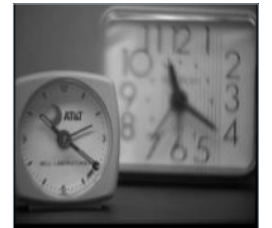

(b)

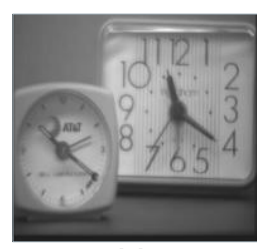

(e)

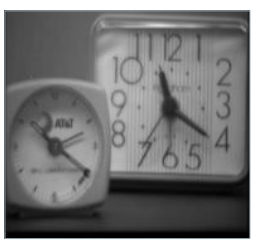

(g)

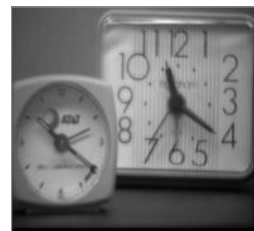

(c)

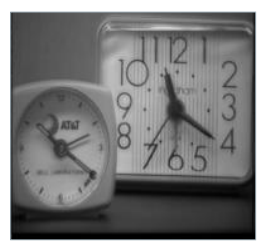

(f)
Fig.4. Fusion results for clock image: (a) left part blurred; (b) right part blurred; (c) fused image by DWT; (d) fused image by PCA; (e) fused image by SWT; (f) fused image by $\mathrm{CBF}$; (g) fused image by proposed method.

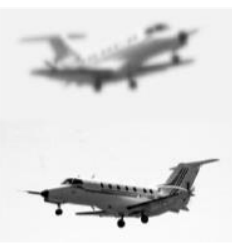

(a)

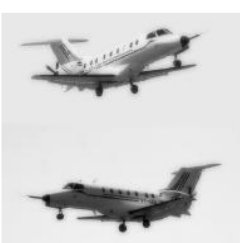

(d)

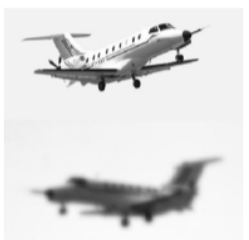

(b)

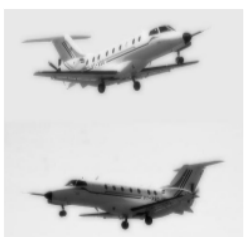

(e)

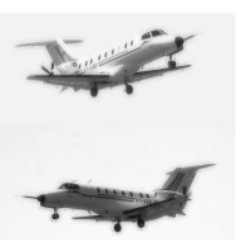

(g)

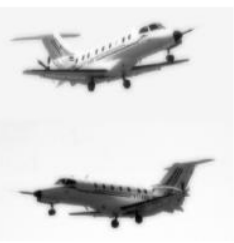

(c)

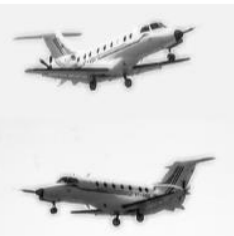

(f)
Fig.5. Fusion results for dataset 1: (a) upper part blurred; (b) lower part blurred; (c) fused image by DWT; (d) fused image by PCA; (e) fused image by SWT; (f) fused image CBF; (g) fused image by proposed method. 


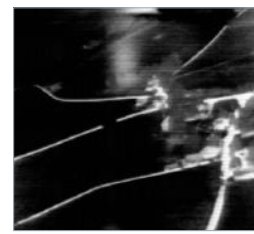

(a)

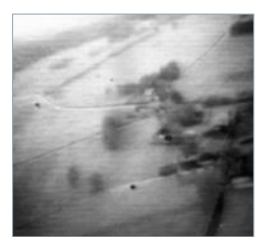

(d)

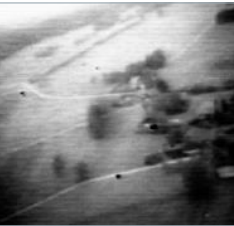

(b)

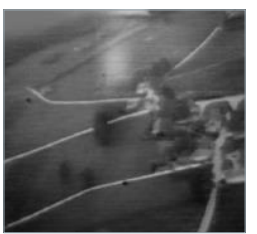

(e)

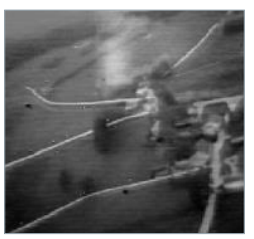

(g)

Fig.6. Fusion results for dataset 2: (a) FLIR image; (b) LLTV image; (c) fused image by DWT; (d) fused image by PCA; (e) fused image by SWT; (f) fused image by CBF; (g) fused image by proposed method.

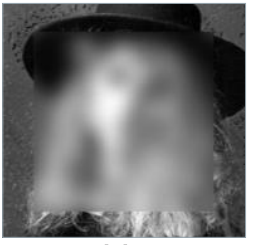

(a)

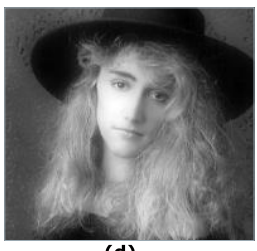

(d)

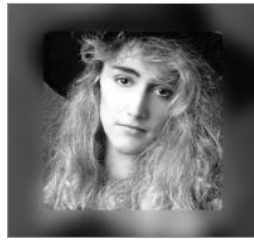

(b)

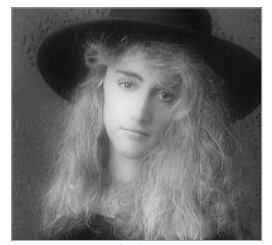

(e)

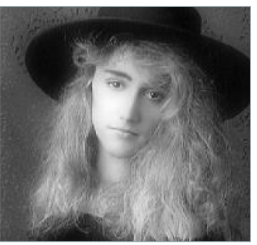

(g)

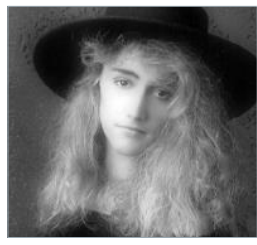

(c)

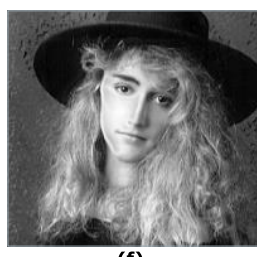

(f)
Fig.7. Fusion results for hoed image: (a) middle part blurred; (b) boundary part blurred; (c) fused image by DWT; (d) fused image by PCA; (e) fused image by SWT; (f) fused image CBF; (g) fused image by proposed method.

Table 2. Image fusion performance measure

\begin{tabular}{|c|c|c|c|c|c|c|c|c|c|c|c|c|c|}
\hline & & \multicolumn{12}{|c|}{ FUSION METRICS } \\
\hline $\begin{array}{l}\text { INPUT } \\
\text { IMAGES }\end{array}$ & $\begin{array}{c}\text { FUSION } \\
\text { METHODS }\end{array}$ & $S D$ & $C C$ & $A P I$ & $A G$ & $E N$ & $M I$ & $F S$ & $S F$ & $Q^{X Y / f}$ & $L^{X Y / f}$ & $N^{X Y / f}$ & $N_{m}^{X Y / f}$ \\
\hline \multirow{5}{*}{ CLOCK } & [8] & 49.315 & 0.9721 & 97.038 & 3.879 & 3.8869 & 5.728 & 1.8904 & 6.2841 & 0.8341 & 0.1659 & 0.0017 & 0.0016 \\
\hline & [26] & 49.315 & 0.9754 & 97.038 & 3.878 & 4.3445 & 5.875 & 1.8722 & 6.2817 & 0.8338 & 0.1662 & 0.0021 & 0.0013 \\
\hline & [12] & 49.408 & 0.9872 & 97.038 & 4.471 & 4.4882 & 6.492 & 1.9021 & 7.4981 & 0.8700 & 0.1299 & 0.0118 & 0.0010 \\
\hline & [24] & 49.892 & 0.9869 & 96.548 & 5.5265 & 7.2755 & 7.3415 & 1.9600 & 10.142 & 0.8982 & 0.0995 & 0.0114 & 0.0011 \\
\hline & PROPOSED & 49.417 & 0.9890 & 97.080 & 4.4044 & 7.2761 & 6.8667 & 1.9934 & 8.0545 & 0.8583 & 0.1406 & 0.0155 & 0.0023 \\
\hline \multirow{5}{*}{ DATASET1 } & [8] & 51.872 & 0.9746 & 221.43 & 3.1869 & 2.5333 & 5.0198 & 1.9587 & 10.2225 & 0.7958 & 0.2042 & 0.0296 & 0.0072 \\
\hline & [26] & 51.873 & 0.9798 & 221.43 & 3.1871 & 2.8173 & 5.0368 & 1.9590 & 10.2232 & 0.7959 & 0.2041 & 0.0279 & 0.0079 \\
\hline & [12] & 52.172 & 0.9876 & 221.43 & 4.2307 & 3.5672 & 5.3640 & 1.9526 & 13.5788 & 0.8698 & 0.1293 & 0.0036 & 0.0052 \\
\hline & [24] & 52.561 & 0.9886 & 220.58 & 4.9215 & 4.2667 & 5.4154 & 1.9635 & 16.9262 & 0.9284 & 0.0635 & 0.0345 & 0.0081 \\
\hline & PROPOSED & 55.242 & 0.9904 & 221.45 & 5.8073 & 4.7166 & 5.0230 & 1.9766 & 18.6560 & 0.8451 & 0.1473 & 0.0285 & 0.0076 \\
\hline \multirow{5}{*}{ DATASET2 } & [8] & 40.394 & 0.5703 & 84.631 & 7.0495 & 1.9383 & 3.0799 & 1.7832 & 10.6120 & 0.6249 & 0.3751 & 0.0173 & 0.0036 \\
\hline & [26] & 41.220 & 0.5770 & 84.631 & 9.7021 & 2.0432 & 3.0765 & 1.7704 & 21.9084 & 0.4599 & 0.5132 & 0.1035 & 0.1182 \\
\hline & [12] & 41.087 & 0.5689 & 84.631 & 9.3246 & 2.0486 & 3.0832 & 1.7543 & 14.0195 & 0.7226 & 0.2753 & 0.0078 & 0.0065 \\
\hline & [24] & 41.358 & 0.5317 & 84.665 & 9.7106 & 7.3465 & 3.0842 & 1.7735 & 16.8049 & 0.7305 & 0.2651 & 0.0184 & 0.0044 \\
\hline & PROPOSED & 41.429 & 0.5815 & 86.642 & 7.7882 & 7.8537 & 3.4738 & 1.8188 & 17.4319 & 0.8310 & 0.1026 & 0.2124 & 0.0021 \\
\hline \multirow{5}{*}{ HOED } & [8] & 56.559 & 0.9521 & 95.951 & 13.2480 & 1.9248 & 4.5982 & 1.8954 & 17.4967 & 0.7168 & 0.2832 & 0.0276 & 0.0028 \\
\hline & [26] & 56.568 & 0.9460 & 95.951 & 13.2807 & 2.0431 & 4.7601 & 1.8643 & 17.5939 & 0.7188 & 0.2812 & 1.0231 & 0.0020 \\
\hline & [12] & 57.947 & 0.9479 & 95.951 & 28.0801 & 7.6941 & 4.7803 & 1.8092 & 30.7982 & 0.8957 & 0.1007 & 0.0254 & 0.0036 \\
\hline & [24] & 58.097 & 0.9628 & 95.958 & 22.8185 & 7.6988 & 4.6434 & 1.9987 & 30.9057 & 0.8775 & 0.1152 & 0.0626 & 0.0072 \\
\hline & PROPOSED & 61.849 & 0.9566 & 96.173 & 25.2017 & 7.7399 & 6.9666 & 1.9952 & 33.9649 & 0.9478 & 0.0483 & 0.0226 & 0.0039 \\
\hline \multirow{5}{*}{$\begin{array}{l}\text { CT AND } \\
\text { MRI }\end{array}$} & [8] & 34.883 & 0.6502 & 32.082 & 5.5005 & 1.6771 & 3.4601 & 1.6204 & 10.2717 & 0.6440 & 0.3560 & 0.0657 & 0.0110 \\
\hline & [26] & 35.155 & 0.6521 & 32.082 & 6.6750 & 1.8607 & 3.4028 & 1.6082 & 13.1607 & 0.6140 & 0.3850 & 0.0052 & 0.0011 \\
\hline & [12] & 35.107 & 0.6246 & 32.082 & 6.1676 & 2.0452 & 3.4122 & 1.6091 & 11.3466 & 0.6914 & 0.3086 & 1.5826 & 0.0021 \\
\hline & [24] & 35.785 & 0.6905 & 32.166 & 7.2431 & 5.9698 & 3.4311 & 1.6554 & 14.7174 & 0.7143 & 0.2843 & 0.0065 & 0.0014 \\
\hline & PROPOSED & 37.862 & 0.6545 & 41.414 & 11.5408 & 6.7736 & 5.5999 & 1.6122 & 21.0958 & 0.9116 & 0.0758 & 0.0873 & 0.0126 \\
\hline
\end{tabular}




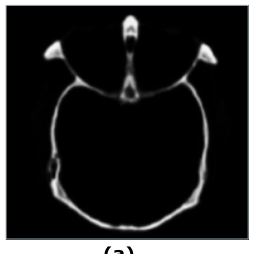

(a)

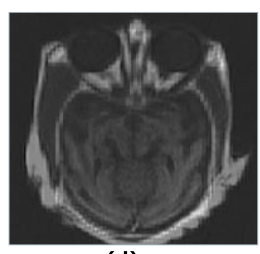

(d)

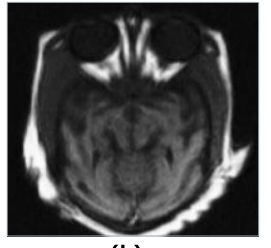

(b)

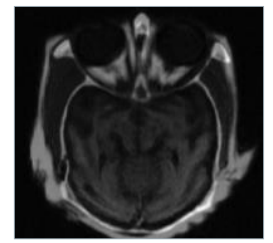

(e)

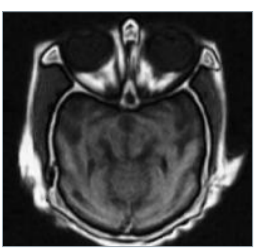

(g)

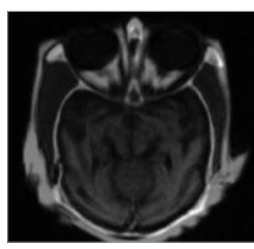

(c)

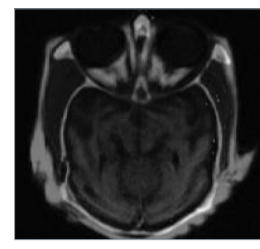

(f)
[5] Slamani M A, Ramac L C, Uner M K, Varshney P K, Weiner D D, Alford M G, Ferris D D, Vannicola V C. Enhancement and fusion of data for concealed weapons detection [J]. Proc SPIE, 1997, 3068: 8-19.

[6] Daniel M M, Willsky A S. A multiresolution methodology for signal-level fusion and data assimilation with applications to remote sensing [J]. Proc IEEE, 1997, 85(1): 164-180.

[7] Mitianoudis N, Stathaki T. Pixel-based and region-based image fusion schemes using ICA bases. Inf. Fusion, 2007, 8 (2): 131-42.

[8] Li H, Manjunath B S, Mitra S K. Multisensor image fusion using the wavelet transform $[\mathrm{J}]$. Graph Models Image Process, 1995, 57(3): 235-245.

[9] Petrovic V. Multisesor pixel-level image fusion. PhD Thesis, Department of Imaging Science and Biomedical Engineering, Manchester School of Engineering, United Kingdom, 2001.

[10] Shrey amsha Kumar B K, Swamy M N S, Omair Ahmad M Multiresolution DCT decomposition for multifocus image fusion. Proceedings of the Canadian Conference on Electrical and Computer Engineering (CCECE), Regina, Canada, 2013, 1-4.

[11] Goshtasby A A, Nikolov S. Image fusion: advances in the state of the art. Inf. Fusion, 2007, 8(2): 114-118.

Fig.8. Fusion results for CT and MRI image: (a) CT image; (b) MRI image; (c) fused image by DWT; (d) fused image by PCA; (e) fused image by SWT; (f) fused image CBF; (g) fused image by proposed method.

\section{CONCLusions}

In this paper, a fusion method of two multi-focus or multisensor images based on DWT and CBF algorithm is presented. In the proposed framework, the average and sharp details of the source images are integrated very cleverly into a single fused image. The performance of the proposed fusion method is tested against other fusion methods using several pairs of test images. Fusion performance is evaluated using many quantitative measure ment criterions. Through the experimental results, it is found that the proposed method preserves more sharp information while eliminating artifacts and has shown better performance than other existing fusion methods visually as well as quantitatively.

\section{ACKNOWLEDGMENT S}

The authors would gratefully acknowledge the support of Dr. V.P.S. Naidu for providing us dataset 1 and dataset2 for the test purpose.

\section{REFERENCES}

[1] Blum R S, Liu Z. Multi-sensor Image Fusion and Its Applications. CRC Press, Tay lor \& Francis Group, 2005.

[2] Hill D, Edwards P, Hawkes D. Review of pixel-level image fusion. Fusing medical images [J]. Image Processing, 1994, 6(2): 22-24.

[3] Qu G H, Zhang D L, Yan P E. Medical image fusion by wavelet transform modulus maxima [J]. Opt Express, 2001, 9(4): 184-190.

[4] Smith M I, Ball A N, Hooper D. Real-time image fusion: A vision aid for helicopter pilotage [J]. Proc SPIE, 2002, 4713: 83-94.
[12] Huafeng Li, Shanbi W, Chai Yi. Multifocus image fusion scheme based on feature contrast in the lifting stationary wavelet domain. EURASIP Journal on Advances in Signal Processing. 2012, 39: 1-16.

[13] Selesnick I W, Baraniuk R G, Kingsbury N C. The dualtree complex wavelet transform. IEEE Signal Process, Mag, 2005, 22 (6): 123-151.

[14] Ali F E, El-Dokany I M, Saad A A, Abd El-Samie F E. A curvelet transform approach for the fusion of MR and CT images. Journal of Modern Optics, Taylor \& Francis. 2010 57 (4): 273-286.

[15] Do M N, Vetterli M. The contourlet transform: an efficient directional multiresolution image representation. IEEE Trans. on Image Processing, 2005, 14(12): 2091-2106.

[16] Zhang Q, Guo B L. Multifocus image fusion using the nonsubsampled contourlet transform. Signal Processing, 2009, 89(7): 1334-1346.

[17] Naidu V P S. Image fusion technique using multiresolution singular value decomposition. Defence Sci. J., 2011, 61 (5): 479-484.

[18] Shutao Li, Xudong Kang, Jianwen Hu, Bin Yang. Image matting for fusion of multi-focus images in dynamic scenes. Inf. Fusion, 2013, 14: 147-162.

[19] Naidu V P S. Discrete cosine transform-based image fusion, Defence Sci. J., 2010, 60 (1): 48-54.

[20] Sonam Gautam, Manoj Kumar. An Effective Image Fusion Technique based on Multiresolution Singular Value Decomposition. INFOCOMP Journal of Computer Science, 2015, 14 (2): 31-43.

[21] Tomasi C, Manduchi R. Bilateral filtering for gray and color images. Proceedings of the Sixth International Conference on Computer Vision, 1998: 839-846.

[22] Manoj Diwakar, Sonam, Manoj Kumar. CT image denoising based on complex wavelet transform using local adaptive thresholding and Bilateral filtering. ACM, Proceedings of the Third International Symposium on Women in Computing and Informatics (WCI), 2015, 297302.

[23] Jianwen $\mathrm{Hu}$, Shutao Li. The multiscale directional bilateral filter and its application to multisensor image fusion. Inf. Fusion, 2012, 13(3): 196-206.

[24] Shreyamsha Kumar B K. Image fusion based on pixel 
significance using cross bilateral filter. SIVIP, 2013.

[25] Petschnigg G, A grawala M, Hoppe H, Szeliski R, Cohen $\mathrm{M}$,Toyama K. Digital photography with flash and no-flash image pairs. ACM Trans. Gr. 2004, 23(3): 664-672.

[26] Naidu V P S, Raol J R. Pixel-level image fusion using wavelets and principle component analy sis. Defence Sci. J., 2008, 58 (3): 338-352.

[27] Li S, Kwok J T, Wang Y. Using the discrete wavelet frame transform to merge Landsat TM and SPOT panchromatic images. Inf. Fusion, 2002, 3(1): 17-23.

[28] Wang Z, Ziou D, Armenakis C, Li D, Li Q. A Comparative Analysis of Image Fusion Methods. IEEE Trans. on Geoscience and Remote Sensing, 2005, 43 (6): $1391-1402$.

[29] Petrovic V, Xydeas C. Objective image fusion performance characterization. In: Proceedings of the International Conference on Computer Vision (ICCV), 2005, 2: 1866-1871.

[30] Eley an Alaa. Enhanced face recognition using data fusion. I. J. Intelligent sy stems and applications, 2013, 01: 98-103.

[31] Kaur S, Dadhwal H S. Biorthogonal wavelet transform using bilateral filter and ad aptive histogram equalization. I. J. Intelligent systems and ap plications, 2015, 03: 37-43.

\section{Authors' Profiles}

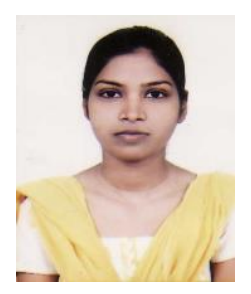

Sonam received the M.C.A. degree in computer science from Babasaheb Bhimrao Ambedkar University, Lucknow, India, in 2012. She is currently working toward the Ph.D. degree at Babasaheb Bhimrao Ambedkar University, Lucknow, India. Her research interest focuses on image fusion and image enhancement.

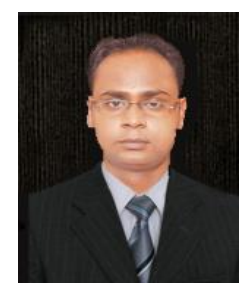

Manoj Kumar received the Ph.D. degree in Computer Vision and Image Processing from Indian Institute of Technology Roorkee, India, in 2011 and M.C.A. degree in computer science from Jawaharlal Nehru University, New Delhi, India, in 2003. He is currently working as Assistant Professor in the Computer Science, Babasaheb Bhimrao Ambedkar University, Lucknow, India. His general research interest include the areas of computer graphics, vision $\&$ image processing, medical imaging, image compression, security and watermarking. He has published various research papers in international and national journals and conferences.

How to cite this paper: Sonam, Manoj Kumar,"Discrete Wavelet Transform and Cross Bilateral Filter based Image Fusion", International Journal of Intelligent Systems and Applications(IJISA), Vol.9, No.1, pp.37-45, 2017. DOI: 10.5815/ijisa.2017.01.04 